\title{
SIGN PRESERVATION PROPERTIES OF SOME NONLINEAR TRANSFORMATIONS
}

\begin{abstract}
SAWINDER P. KAUR
Abstract. It is shown that the number of sign changes in certain transformations does not increase with each iteration in time. These transformations are composed of linear components defined in terms of totally positive matrices and semi linear components similar to $u \rightarrow k u^{3}$. In particular the analysis shows that for certain semi linear parabolic equations discretized using finite difference methods, the number of sign changes does not increase.
\end{abstract}

Mathematics subject classification (2010): Primary 47J05; Secondary 47J25, 15A90, 65N06.

Keywords and phrases: Nonlinear transformation, finite difference method, totally positive matrix.

\section{REFERENCES}

[1] Richard L. Burden, J. Douglas Faires, Numerical Analysis, eight edition, Thomson Books, 2005.

[2] F. Dalfovo, S. Giorgini, L.P. Pitaevskit, S. Stringari, Theory of Bose-Einstein condensation in trapped gases, Reviews of Modern Physics, 71, 3 (1999), 463-512.

[3] J.W. Demmel, Applied Numerical Linear Algebra, SIAM, 1997.

[4] M. Gasca, J.M. Pe $\tilde{n}$ A, On Factorizations of Totally Positive Matrices, Linear Algebra Application, 165 (1992), 25-44.

[5] S. Karlin, Total Positivity, Stanford University Press, April 1968.

[6] P. Lancaster And M. Tismenetsky, The Theory of Matrices, Second Edition with applications, Academic Press, 1985.

[7] J. Stoer, And R. Bulrisch, Introduction to Numerical Analysis, Springer-Verlag New York, 1980.

[8] M. TABATA, A Finite Difference Approach to the Number of Peaks of Solutions for Semilinear Parabolic Problems, Journal of Mathematical Society of Japan, 32, 1 (1980), 171-191. 